\title{
Significant Muscle Hemorrhage Associated With Low-Molecular-Weight Heparin Use in Dermatomyositis: A Case Report
}

\author{
Jin Young Lee ${ }^{\mathrm{a}}$, Jehun Kimª, b
}

\begin{abstract}
Dermatomyositis is a rare systemic inflammatory disease with characteristic skin features and muscular involvement. Few cases of hemorrhagic myositis with dermatomyositis have been reported worldwide. Here, we report a case of a 43-year-old man with dermatomyositis, which was treated with steroids and low-molecular-weight heparin. During the treatment course, he complained of acute left thigh pain. Computed tomography of the lower extremity showed hemorrhage in the left iliopsoas, iliacus, thigh muscle, and retroperitoneum. We discontinued heparin treatment and applied a splint with bed rest. U1timately, he had a successful recovery. Patients with dermatomyositis may have an intrinsic risk of life-threatening muscle hemorrhage, and anticoagulation treatment may induce significant muscle hemorrhage in such patients.
\end{abstract}

Keywords: Dermatomyositis; Hemorrhage; Low-molecular-weight heparin; Iliopsoas muscle; Thigh muscle

\section{Introduction}

Dermatomyositis (DM) is an autoimmune idiopathic inflammatory myopathy with characteristic skin features such as heliotrope rash, $\mathrm{V}$ sign, shawl sign, and holster sign, which are present on the face and other sites, including the back and extremities [1]. Symptoms of DM may include restrictive lung disease, interstitial lung disease, pneumomediastinum, myositis, arthritis, mechanic's hands, cardiomyopathy, and fever [1,2].

There have been few reports of DM causing hemorrhagic myositis [3-7]. We present a case of hemorrhagic myositis associated with the use of low-molecular-weight heparin (LMWH) in DM. The patient was treated for dermatomyositis

Manuscript submitted August 12, 2019, accepted September 7, 2019

aDepartment of Internal Medicine, Kosin University Gospel Hospital, Kosin University College of Medicine, Busan, Korea

${ }^{\mathrm{b} C}$ Corresponding Author: Jehun Kim, Department of Internal Medicine, Kosin University College of Medicine, 262, Gamcheon-ro, Seo-gu, Busan 602-702, Korea. Email: libertier@gmail.com

doi: https://doi.org/10.14740/jmc3354 with suspected pulmonary vein embolism but developed spontaneous hemorrhage of the left iliopsoas and iliacus muscles.

To the best of our knowledge, few cases of DM accompanied by hemorrhagic myositis have been reported worldwide to date, and none have been reported in Korea. Therefore, we describe the first Korean case report of DM with hemorrhagic myositis associated with LMWH.

\section{Case Report}

The patient was a 43 -year-old Korean man. His occupation was welding, and his medical history was clear. In February 2015, he developed fever with headache and visited a local hospital, where he was admitted. There he developed right supraorbital edema and cramping pain in both legs. Laboratory findings were as follows: D-dimer $395 \mathrm{ng} / \mathrm{mL}$, aspartate aminotransferase (AST) $412 \mathrm{IU} / \mathrm{L}$, alanine amino transferase (ALT) 217 IU/L, and creatine phosphokinase (CPK) 1,610 IU/L. Immunofluorescence assay was negative for antinuclear antibodies (ANAS), serum anti-Sm/RNP, anti-neutrophil cytoplasmic antibodies, anti-SSa and anti-SSb antibodies, anti-cardiolipin antibodies, anti-dsDNA antibodies, and anti-Jo-1 antibodies. Chest radiography showed haziness in both lower lobes. Computed tomography (CT) showed multifocal peribronchial consolidation and ground-glass opacity in both lower lobes, especially in the right subpleural area, and effusion. A filling defect $(1.5 \mathrm{~cm}$ in diameter) was present in the distal right lower pulmonary vein (Fig. 1). Oral moxifloxacin $400 \mathrm{mg}$ and intravenous methylprednisolone $125 \mathrm{mg}$ were administered for 4 days, which slightly improved his symptoms.

Eventually, he was referred to our hospital in March 2015, where he presented with continued fever and headache. On admission, his height was $173 \mathrm{~cm}$, body weight was 54.2 $\mathrm{kg}$, body temperature was $37.3^{\circ} \mathrm{C}$, and other vital signs were normal. He exhibited DM-associated skin rashes, a heliotrope rash and edema on both eyelids and eruptions on the knuckles of both hands, the prepatellar areas, both shoulders, and the back (Fig. 1).

Laboratory findings were as follows: white blood cell count, 6,310/ $\mu \mathrm{L}$; hemoglobin, $13.9 \mathrm{~g} / \mathrm{dL}$; platelets, 177,000/ $\mu \mathrm{L}$; CPK, 293 IU/L; AST, 90 IU/L; ALT, 70 IU/L; and aldolase, 14.0 U/L. Para nasal sinuses (PNS) film showed fluid level in the right maxillary antrum, indicative of acute sinusitis or blood accumulation in the right maxillary antrum. Neurologi- 

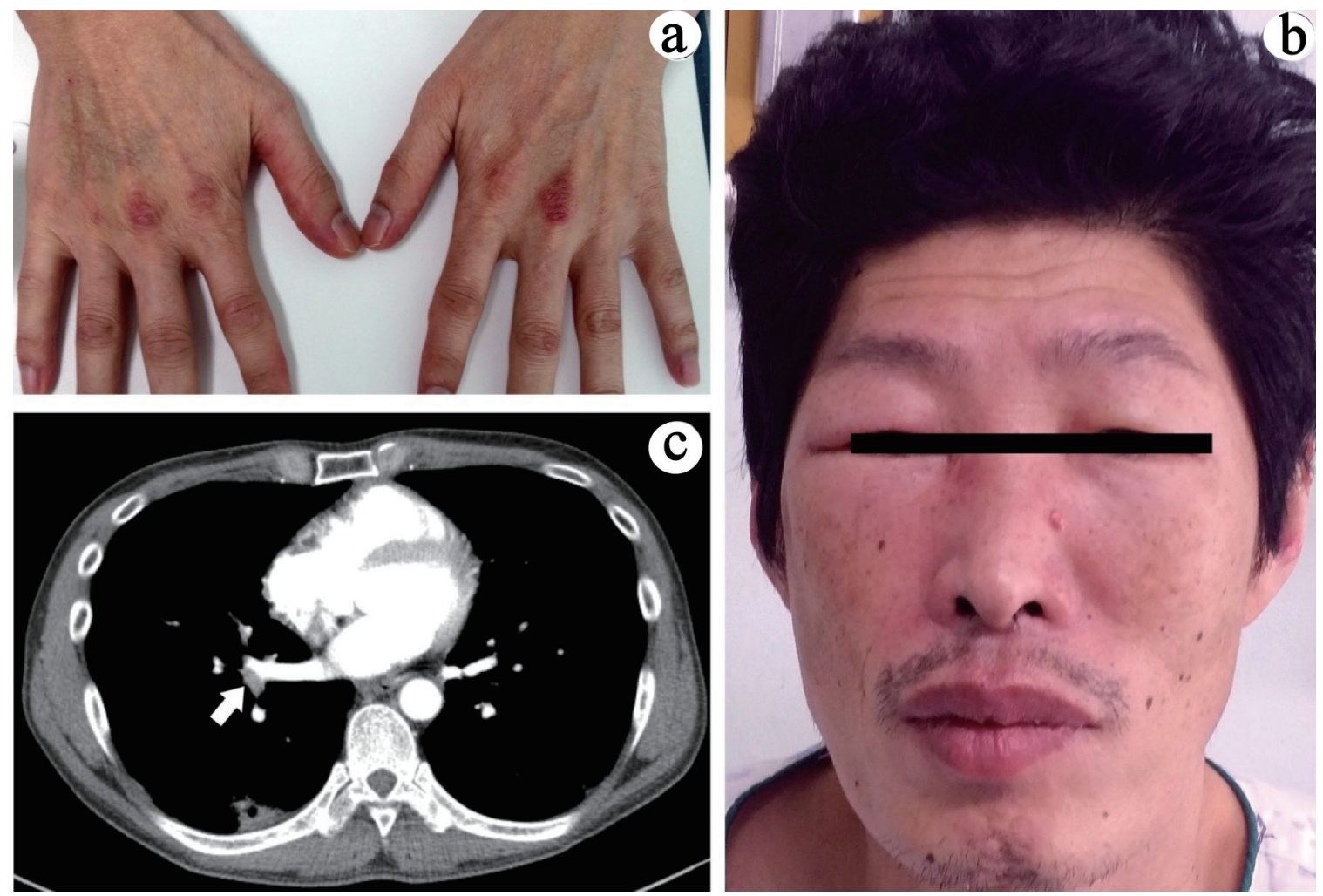

Figure 1. (a) Gottron's papules on both hands. (b) Both periorbital edema and heliotrope rash. (c) Focal wedge-shaped consolidation in right lower lobe and filling defect in the distal right lower pulmonary vein (arrow).

cal examination showed no motor weakness. An electromyogram showed no definitive evidence of myopathy or polyneuropathy. Skin punch biopsies of rashes on his left hand, left knee, and right back revealed superficial and deep perivascular dermatitis.

Collectively, these symptoms led to a diagnosis of amyopathic DM with suspected right pulmonary vein thromboembolism [8]. Intravenous methylprednisolone (125 mg once a day) and subcutaneous enoxaparin (5,000 IU twice a day) were administered. Cutaneous symptoms and laboratory findings improved gradually.

After 10 days of treatment, the patient developed dyspnea, tachycardia, and left thigh pain. His hemoglobin decreased from 13.7 to $7.3 \mathrm{~g} / \mathrm{dL}$ and blood pressure fell to $90 / 60 \mathrm{~mm} \mathrm{Hg}$. Platelets, prothrombin time (PT), activated partial thromboplastin time (APTT), and bleeding time (BT) were all normal. A CT scan showed hemorrhage of the left iliopsoas, iliacus, and retroperitoneum (Fig. 2). LMWH treatment was discontinued, and three units of packed red cells were transfused. We applied a splint on his lower extremities and adviced complete bed rest. He then recovered successfully and was discharged on 25 days after admission.

\section{Discussion}

The occurrence of hemorrhagic myositis associated with DM on heparin treatment is a rare but potentially fatal situation. To the best of our knowledge, only few cases have been reported [3-7]. We analyzed these six patients including our case (Table 1 [3-7]). Case 1: an 80-year-old man was diagnosed with DM. He was treated with intravenous methylprednisolone followed by oral prednisone. Prophylactic treatment with unfractionated heparin (UFH) was also initiated. Although APTT was slightly prolonged, at $42 \mathrm{~s}, \mathrm{PT}$ and BT were normal. After 10 days, CT showed left rectus sheath and oblique right thigh muscle hemorrhage. Case 2: a 77-year-old woman was diagnosed with DM with interstitial pneumonia. She was treated with intravenous methylprednisolone followed by oral prednisone. After 2 weeks, she reported chest wall pain. A diagnosis of unstable angina was made, and a daily regimen of UFH, ticlopidine, and aspirin was initiated. After 4 days, hemorrhage of the left iliopsoas, iliacus, and retroperitoneum was found. APTT was prolonged and PT was normal. Case 3: a 64-year-old woman was diagnosed with DM with rapidly progressive interstitial pneumonia. She was treated with steroids, cyclosporin A, cyclophosphamide and LMWH (dalteparin). On day 10, she complained of lower abdominal pain. CT showed hemorrhage in the right psoas and iliacus, retroperitoneum, and left rectus sheath. APTT and PT were normal. Case 4: a 65-year-old woman was diagnosed with DM. She was treated with intravenous methylprednisolone for 3 days, followed by oral prednisolone. Treatment with prophylactic UFH was initiated. After 4 days, APTT was prolonged, at $>100 \mathrm{~s}$; PT was normal. CT showed hemorrhage of the iliopsoas muscle on both sides and the thigh muscles. Case 5: a 60-year-old man was diag- 

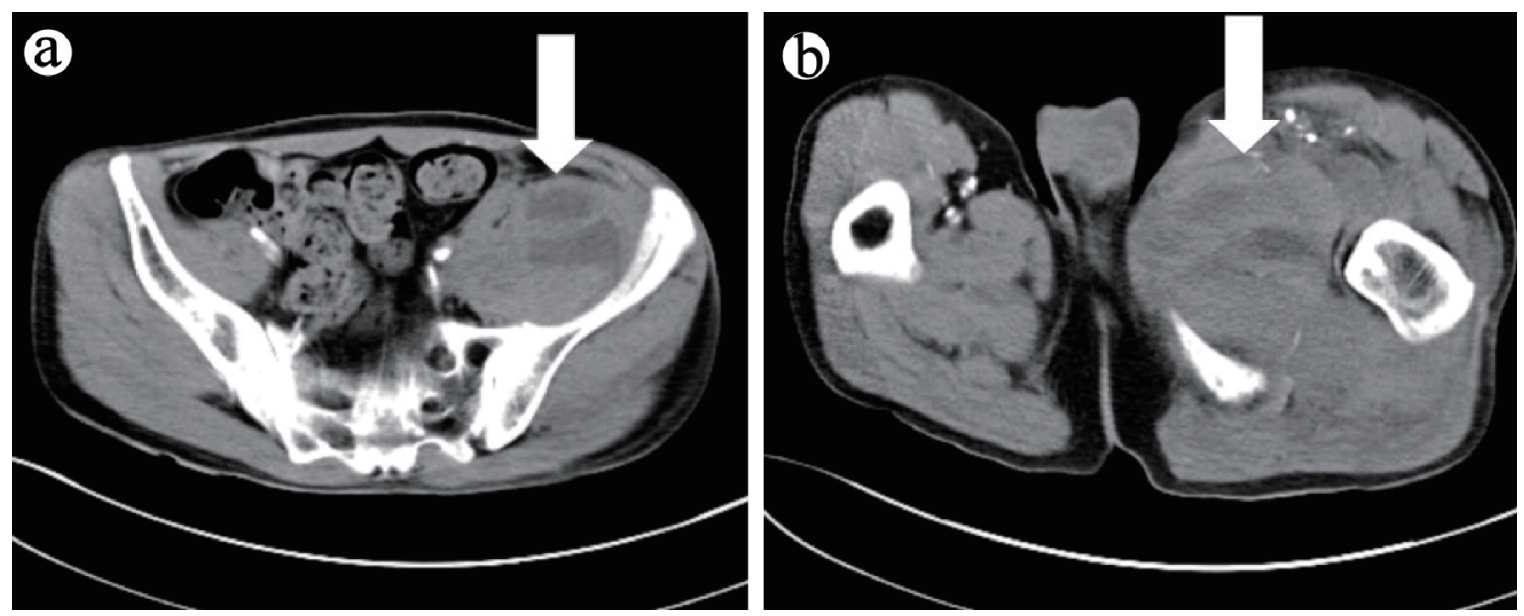

Figure 2. CT scan of the lower extremities after bleeding. CT scan with contrast shows bleeding into (a) iliopsoas muscle (arrow), and (b) thigh muscle (arrow). CT: computed tomography.

nosed with DM. He was treated with oral prednisolone. On day 25, he developed respiratory failure. An intravenous pulse of methylprednisolone and UFH were initiated. After 6 days of treatment, CT showed intramuscular hemorrhage from the left deltoid. APTT was prolonged at $89 \mathrm{~s}$. UFH was discontinued. However, after 3 days, a second hemorrhage occurred despite normal coagulability. CT showed another intramuscular hematoma in the trapezius muscle.

Orrel et al [9] presented two cases in which hemorrhage in patients with DM was presumed to be related to trauma or vessel wall fragility caused by capillary vasculitis, because patients did not have a bleeding diathesis and heparin was not used. However, recently, muscle hemorrhage was found in a patient receiving anticoagulant therapy. Heparin treatment results in an increased risk of major bleeding [10]. The use of LMWH can lead to major complications, such as acute abdominal hemorrhage, which can be life-threatening [11].

In four of the six cases discussed here, bleeding occurred in the iliopsoas muscle. Patients who are anticoagulated with heparin are at an increased risk of developing an iliacus or psoas hematoma, manifesting in a wide range of symptoms from groin pain to massive bleeding and shock [12]. Identification of these occurrences is crucial in patients with DM.

In patients with DM, capillary depletion occurs and capillary density is significantly decreased. These capillary changes are not present in patients with polymyositis. We believe that muscle hemorrhage in DM is related to vessel fragility due to capillary vasculitis, muscle tissue weakness caused by steroid treatment of DM, and the use of anticoagulant agents.

Here, we report a case of DM complicated with spontaneous intramuscular hemorrhage while on heparin. This case and others suggest that when treating a patient with DM, we need to think twice about heparin therapy.

DM is a well-known condition that represents acquired and potentially treatable causes of skeletal muscle weakness. However, DM with hemorrhagic myositis is a rare condition, with few cases having been reported worldwide and none reported in Korea. Here, we reported a rare case of DM accom-

Table 1. Comparison of Six Cases of Dermatomyositis Accompanied by Hemorrhagic Myositis With Heparin Use

\begin{tabular}{|c|c|c|c|c|c|c|c|c|c|}
\hline Case & Age & Sex & Bleeding sites & $\begin{array}{l}\text { Type of } \\
\text { anticoagulant }\end{array}$ & $\begin{array}{l}\text { Onset after } \\
\text { administration } \\
\text { of anticoagulant }\end{array}$ & Coagulability & Treatment & Outcome & Reference \\
\hline 1 & 80 & M & $\begin{array}{l}\text { Left rectus sheath, } \\
\text { oblique, right thigh }\end{array}$ & $\mathrm{UFH}$ & 9 days & APTT prolonged & Transfusion & Alive & {$[3]$} \\
\hline 2 & 77 & $\mathrm{~F}$ & $\begin{array}{l}\text { Left iliopsoas, left } \\
\text { iliacus, retroperitoneum }\end{array}$ & UFH, ticlopidine & 4 days & APTT prolonged & Embolization & Death & {$[4]$} \\
\hline 4 & 65 & $\mathrm{~F}$ & Both iliopsoases, thighs & UFH & 4 days & APTT prolonged & Transfusion & Alive & {$[6]$} \\
\hline 5 & 60 & M & Left deltoid, left trapezius & UFH & 6 days & APTT prolonged & Transfusion & Death & {$[7]$} \\
\hline 6 & 43 & M & $\begin{array}{l}\text { Left iliopsoas, left } \\
\text { iliacus, retroperitoneum }\end{array}$ & Enoxaparin & 10 days & Normal & Transfusion & Alive & Our case \\
\hline
\end{tabular}

M: male; F: female; UFH: unfractionated heparin; APTT: activated partial thromboplastin time. 
panied by hemorrhagic myositis with the use of LMWH. We expect that our case report will inform clinicians of the risk of using heparin in patients with inflammatory myopathy.

\section{Acknowledgments}

The patient was treated at Kosin University Gospel Hospital, Busan, South Korea.

\section{Financial Disclosure}

None to declare.

\section{Conflict of Interest}

None to declare.

\section{Informed Consent}

Informed consent was obtained from the patient for publication of this case report and any accompanying images.

\section{Author Contributions}

Both authors contributed to the writing and editing of this manuscript as well as being involved in the patient treatment.

\section{References}

1. Bohan A, Peter JB. Polymyositis and dermatomyositis (first of two parts). N Engl J Med. 1975;292(7):344-347.

2. Katzap E, Barilla-LaBarca ML, Marder G. Antisynthetase syndrome. Curr Rheumatol Rep. 2011;13(3):175-181.

3. Langguth DM, Wong RC, Archibald C, Hogan PG. Haemorrhagic myositis associated with prophylactic heparin use in dermatomyositis. Ann Rheum Dis. 2004;63(4):464-465.

4. Higashi Y, Mera K, Kanzaki T, Kanekura T. Fatal muscle haemorrhage attributable to heparin administration in a patient with dermatomyositis. Clin Exp Dermatol. 2009;34(3):448-449.

5. Yamagishi M, Tajima S, Suetake A, Kawakami H, Watanabe T, Kuriyama H, Takada T, et al. Dermatomyositis with hemorrhagic myositis. Rheumatol Int. 2009;29(11):13631366.

6. Hanawa $\mathrm{F}$, Inozume $\mathrm{T}$, Harada $\mathrm{K}$, Andou N, Kawamura T, Shibagaki N, Shimada S. A case of dermatomyositis accompanied by spontaneous intramuscular hemorrhage despite normal coagulability. Rheumatol Int. 2013;33(11):2949-2950.

7. Miwa Y, Muramatsu M, Takahashi R, Wakabayashi K, Odai T, Isozaki T, Yajima N, et al. Dermatomyositis complicated with hemorrhagic shock of the iliopsoas muscle on both sides and the thigh muscle. Mod Rheumatol. 2010;20(4):420-422.

8. Sontheimer RD. Cutaneous features of classic dermatomyositis and amyopathic dermatomyositis. Curr Opin Rheumatol. 1999;11(6):475-482.

9. Orrell RW, Johnston HM, Gibson C, Cass RM, Griggs RC. Spontaneous abdominal hematoma in dermatomyositis. Muscle Nerve. 1998;21(12):1800-1803.

10. Wada Y, Yanagihara C, Nishimura Y. Bilateral iliopsoas hematomas complicating anticoagulant therapy. Intern Med. 2005;44(6):641-643.

11. Di Ascenzo L, Cassin M, Driussi M, Moretti M, Pecoraro R, Nicolosi GL. Major rectus abdominis hematoma complicating low molecular weight heparin therapy. J Cardiovasc Med (Hagerstown). 2008;9(7):758-759.

12. Sasson Z, Mangat I, Peckham KA. Spontaneous iliopsoas hematoma in patients with unstable coronary syndromes receiving intravenous heparin in therapeutic doses. Can J Cardiol. 1996;12(5):490-494. 